\title{
L-DWARF BINARIES IN THE 20-PARSEC SAMPLE*
}

\author{
I. Neill Reid ${ }^{1}$, K. L. Cruz ${ }^{2,5}$, Adam J. Burgasser ${ }^{3}$, And Michael C. Liu ${ }^{4,6}$ \\ ${ }^{1}$ Space Telescope Science Institute, 3700 San Marin Drive, Baltimore, MD 21218, USA; inr@stsci.edu \\ ${ }^{2}$ Department of Astronomy, MC 105-24, California Institute of Technology, Pasadena, CA 91125, USA; kelle@ astro.caltech.edu \\ ${ }^{3}$ Massachusetts Institute of Technology, Kavli Institute for Astrophysics and Space Research, Building 37, Room 664B, 77 Massachusetts Avenue, \\ Cambridge, MA 02139, USA; ajb@mit.edu \\ ${ }^{4}$ Institute for Astronomy, University of Hawaii, Honolulu, HI 96822, USA; mliu@ifa.hawaii.edu \\ Received 2007 October 4; accepted 2007 November 15; published 2008 January 15
}

\begin{abstract}
We have used the NICMOS NIC1 camera on the Hubble Space Telescope to obtain high-resolution near-infrared images of 27 nearby ultracool dwarfs with formal distance estimates less than 20 parsecs. One target, 2MASS $\mathbf{J} 22551861-5713056$, is resolved as a binary system, with a 4:1 flux ratio in the F110W passband. The primary has spectral type L6, and the revised spectroscopic parallax places it at a distance of $\sim 12$ parsecs. The secondary is likely to have a spectral type between L8 and T1. The remaining 26 dwarfs are unresolved. Combining the present results with our earlier NICMOS (Near-Infrared Camera and Multi Object Spectrometer) observations and literature data, high-resolution imaging exists for 72 of the $85 \mathrm{~L} \mathrm{dwarfs} \mathrm{in} \mathrm{the} \mathrm{current} \mathrm{20-parsec} \mathrm{census.} \mathrm{We} \mathrm{derive} \mathrm{a} \mathrm{formal} \mathrm{frequency}$ of $15.3_{-3.3}^{+5.1} \%$ for resolved binaries in the local L-dwarf population. The frequency drops to $12.5_{-3.0}^{+5.3} \%$ if we take Malmquist bias into account. The NICMOS observations are capable of detecting systems with mass ratios $q>0.2$, but all known binaries are near equal-mass, suggesting that low- $q$ systems either fail to form, or have short lifetimes.
\end{abstract}

Key words: binaries: visual - stars: low-mass, brown dwarfs

\section{INTRODUCTION}

The statistical properties of binary systems provide key insight into the star-formation process. Considerable progress has been made in discerning systematic behavior patterns, notably as a function of mass, since the first thorough investigations by Duquennoy \& Mayor (1991) and Fischer \& Marcy (1992). Recent observations have confirmed the initial indications that binary fraction decreases as a function of mass. The reduced frequency stems at least partly from a decrease in the maximum separation of binaries as a function of total mass (Reid et al. 2001; Burgasser et al. 2003). There is also a clear tendency toward equal-mass binary systems at lower masses. These features may be related to the lower binding energy of those systems, as discussed by Close et al. (2003) and Burgasser et al. (2007a).

Over the last five years, we have been using data from the 2 Micron All Sky Survey (2MASS, Skrutskie et al. 2006) to conduct a census of ultracool ${ }^{7}$ dwarfs in the immediate Solar Neighborhood. The near-infrared photometry allows us to select candidate late-type $\mathrm{M}$ and $\mathrm{L}$ dwarfs, and we estimate distances using spectroscopic parallaxes derived from follow-up optical and near-infrared spectroscopy (Cruz et al. 2003, 2007). Based on those techniques, we have compiled a catalog of $85 \mathrm{~L}$ dwarfs with formal distance estimates less than 20 parsecs (Reid et al. 2007).

As part of this program, we have been using the Hubble Space Telescope (HST) to obtain high-angular-resolution imaging of nearby ultracool dwarfs, searching for resolved binary systems. Reid et al. (2006a) describe observations of 50 dwarfs, including

\footnotetext{
* Based on observations made with the NASA/ESA Hubble Space Telescope, obtained from the Space Telescope Science Institute, which is operated by the Association of Universities for Research in Astronomy, Inc., under NASA contract NAS 5-26555.

5 Spitzer Fellow.

6 Alfred P. Sloan Research fellow.

7 Ultracool dwarfs are defined as dwarfs with spectral types M7 or later i.e. M7-M9.5, L or T (Kirkpatrick et al. 1997).
}

$47 \mathrm{~L}$ dwarfs and three late-type M dwarfs; $44 \mathrm{~L}$ dwarfs from that dataset are in the 20-parsec sample. Here, we present NICMOS (Near-Infrared Camera and Multi Object Spectrometer) imaging of a further 27 ultracool dwarfs, including four late-type M and $23 \mathrm{~L}$ dwarfs; $21 \mathrm{~L}$ dwarfs are in the 20-parsec sample. The following section describes the observations, and Section 3 presents the results, with particular discussion of a new binary system discovered through our observations. In Section 4, our results are combined with literature measurements of a further $7 \mathrm{~L}$ dwarfs, providing data for 72 of the 85 systems currently placed within 20 parsecs of the Sun. The final section summarizes our conclusions.

\section{OBSERVATIONS AND DATA REDUCTION}

The ultracool $\mathrm{M}$ and $\mathrm{L}$ dwarfs discussed in this paper were observed in the course of the Cycle 15 HST Snapshot program (GO 10879). As discussed in Section 1, the targets were selected on the basis that their distances are estimated as less than 20 parsecs. Only a handful, however, have accurate trigonometric parallaxes, and in most cases the distance estimates are based on the spectral-type/ $M_{J}$ relation defined by Cruz et al. (2003). For a few dwarfs, more recent observations have led to revised spectral types, and hence revised distances.

Observations of 30 targets were scheduled in Cycle 15 , but only 28 were successful. In one case, 2MASS J1010148-040649, the observations suffered from a guide star failure; in the second, 2MASS J0857585+570851, the target fell outside the NIC1 field of view. We have determined that the proper motions derived by Schmidt et al. (2007) for the latter source are incorrect; the correct motions are $\mu \sim 0.25^{\prime \prime} \mathrm{yr}^{-1}$, $\theta \sim 270^{\circ}$, rather than $\theta \sim 90^{\circ}$. Observations of one target, 2M2224-0158, an unresolved L4.5 dwarf, are discussed in Reid et al. (2006a), so the present paper discusses new data for 27 ultracool dwarfs.

The successful observations follow the scheme outlined in Reid et al. (2006a). All targets were observed using the NIC1 camera and the F110W and F170M filters. Both sets 


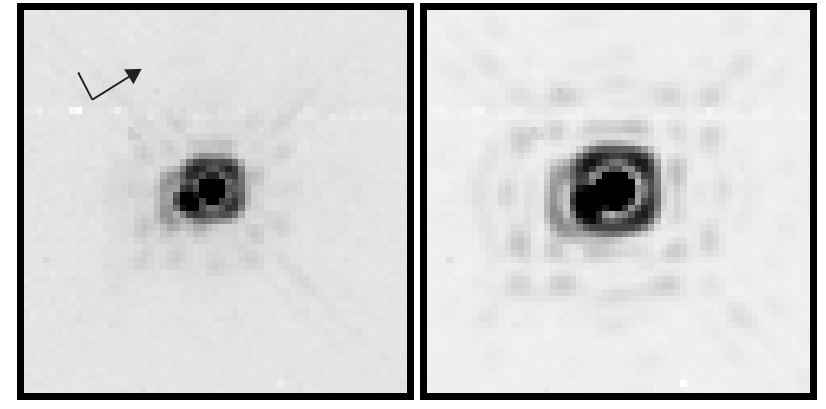

Figure 1. NICMOS images of the L-dwarf binary system, 2M2255-5713. The left-hand panel shows the F110W data, and indicates the cardinal directions; the right-hand panel plots the F170M data.

of observations consist of a pair MULTIACCUM exposures, nodding 2.0 arcsec between the two exposures, with total exposure times of $284 \mathrm{~s}$ at $\mathrm{F} 110 \mathrm{~W}$ and $896 \mathrm{~s}$ in the F170M filter. The combined data give limiting magnitudes of $m_{110} \sim 21.9$ and $m_{170} \sim 20.0 \mathrm{mag}$ (on a Vega-based magnitude system) for isolated point sources. For L dwarfs, these limits correspond to $J \sim 21.2$ and $H \sim 20.0$ (Reid et al. 2006b). Later-type T dwarfs have blue $\left(m_{110}-m_{170}\right)$ colors, $<0.4$ mag (see Figure 4 in Reid et al. 2006b); thus, the F110W data offer greater potential for the detection of faint late-type T-dwarf companions.

The individual NICMOS dataframes were processed and combined to form a single image of each object in the F110W and F170 passbands using the HST pipeline. We used standard IRAF routines ${ }^{8}$ to analyze the final images. The data have a plate scale of 0.043 arcsec pixel ${ }^{-1}$, and the angular resolution is 0.09 arcsec in F110W and 0.14 arcsec in F170M. Thus, the higher angular resolution also favors the F110W images as providing the stronger limits on binary frequency.

\section{ANALYSIS AND RESULTS}

We have analyzed the near-infrared images using the same techniques described in Reid et al. (2006a). First, we used visual inspection to identify obvious close binary systems. Second, we used the imexam and daophot routines in IRAF to determine the full-width half-maxima of the point-spread function (PSF), searching for sources with unusual profiles. Finally, we have used image subtraction to eliminate (as far as possible) the Airy disk, probing for close, faint companions.

The results are straightforward. As in our previous analysis, we have not identified any very low luminosity (late-type $\mathrm{T}$ dwarf) companions associated with the ultracool dwarfs in our sample. Twenty-six sources are unresolved in the NICMOS observations. We used aperture photometry to determine magnitudes for those sources, and Table 1 lists those data, together with 2MASS JHK photometry and astrometry.

The only confirmed binary system in the present sample is the late-type L dwarf, 2MASS J2255186-571305 (Reid et al. 2007), resolved with a separation of $\sim 0.12 \mathrm{arcsec}, \theta=180^{\circ}$ and a flux ratio of 4:1 in the F110W filter (Figure 1). The remaining targets show no evidence for the presence of ultracool companions, although we note that the M9.5 dwarf, $2 \mathrm{M} 2237+3922$, is itself a wide companion (separation 33.6 arcsec, or $635 \mathrm{AU}$ ) of

\footnotetext{
8 IRAF is distributed by the National Optical Astronomy Observatories, which are operated by the Association of Universities for Research in Astronomy, Inc., under cooperative agreement with the National Science Foundation.
}

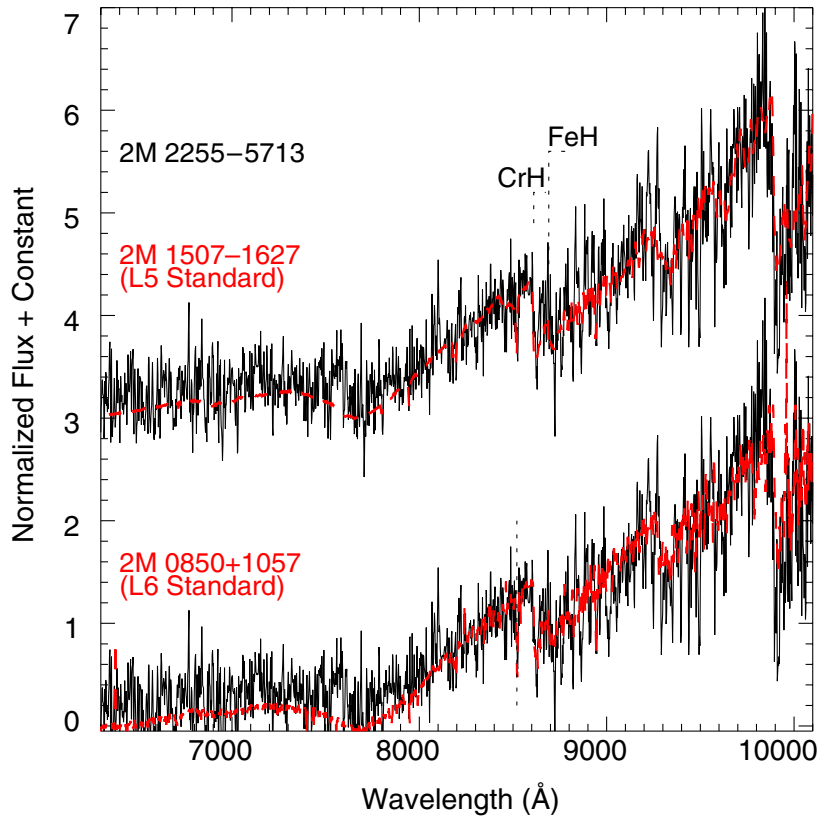

Figure 2. Far-red optical spectrum of 2M2255-5713 matched against the L6 standard, 2M0850+1057, and the L5 standard, 2M1507-1627. The 2M2255-5713 data are noisy at shorter wavelengths, but there is closer agreement with $2 \mathrm{M} 2255-5713$ in the depth of the $\mathrm{CrH}$ and $\mathrm{FeH}$ features at $8600 \AA$ indicating an optical spectral type of $\mathrm{L} 6 \pm 1$.

G216-7, itself an M3.5/M3.5 binary (Kirkpatrick et al. 2001). Table 1 lists the photometric properties of those systems, including $m_{110}$ and $m_{170}$ mag on the Vega-based magnitude system.

We obtained optical spectroscopy of 2M2255-5713 in the course of our 2MASS ultracool survey (Cruz et al. 2003, 2007), the survey that forms the basis for the 20-parsec census. The observations were made on 2004 August 9 using the $\mathrm{RC}$ spectrograph on the 4-metre telescope at Cerro Tololo Interamerican Observatory, and full details on the data reduction techniques are given in Cruz et al. (2007). The resultant spectrum, shown in Figure 2, has only moderate signal-to-noise at shorter wavelengths. However, a comparison with data for the L6 standard, 2M0850+1057 (Kirkpatrick et al. 1999), shows good agreement in the depth of the $\mathrm{CrH}$ and $\mathrm{FeH}$ bands at $8600 \AA$ indicating a spectral type of L6 \pm 1 . This typing is consistent with the analysis of Kendall et al. (2007) of the nearinfrared spectrum, where they estimate a spectral type of L5/L6.

As a single $\mathrm{L}$ dwarf, the spectroscopic parallax for 2M2255-5713 gives a formal distance estimate of $10.8 \pm 3.0$ parsecs. The combined photometry, from 2MASS measurements, gives measurements of $J=14.08, H=13.19$ and $K_{S}=12.58$. The flux ratio measured for the two components in the F110W filter is $4: 1$, implying $\Delta J \sim 1.5 \mathrm{mag}$ and apparent magnitudes of $J \sim 14.3$ for the primary and $J \sim 15.8$ for the fainter secondary. In the F170M filter, the component flux ratio is 3.5:1, corresponding to $\Delta H \sim 1.35$ and $H$-band magnitudes of $H_{1} \sim 13.45$ and $H_{2} \sim 14.8$, or $(J-H)$ colors of 0.85 and 1.0 mag, respectively.

Adopting a spectral type of L6 for the primary, the $M_{J} /$ spectral type relation implies a revised distance estimate of $11.6 \pm 3.1$ parsecs; a spectral type of L5 corresponds to $M_{J} \sim 13.6$ and a distance of $14.3 \pm 3.8$ parsecs. The observed angular separation therefore corresponds to a linear separation between 1.4 and 1.7 AU. The secondary is intrinsically very 
Table 1

Unresolved Ultracool Dwarfs Observed in this Program

\begin{tabular}{|c|c|c|c|c|c|c|c|c|}
\hline 2MASS name & Sp. type & $J$ & $H$ & $K_{S}$ & $m_{110}$ & $m_{170}$ & $d$ (parsec) & Notes \\
\hline 2MASS J002424.6-015819 & M9.5 & 11.86 & 11.12 & 10.58 & 12.45 & 11.11 & $11.55 \pm 0.53$ & BRI0021, T95, 1 \\
\hline 2MASS J010921.7+294925 & M9.5 & 12.91 & 12.16 & 11.68 & 13.53 & 12.19 & $18.3 \pm 1.1$ & 2 \\
\hline 2MASS J022842.4+163933 & L0 & 13.17 & 12.33 & 11.82 & 13.75 & 12.37 & $19.4 \pm 2.3$ & 3 \\
\hline 2MASS J025114.8-035245 & L3 & 13.08 & 12.26 & 11.65 & 13.73 & 12.27 & $12.7 \pm 1.1$ & 4, B07 \\
\hline 2MASS J025503.5-470050 & L8 & 13.23 & 12.19 & 11.53 & 13.97 & 12.22 & $4.9 \pm 0.7$ & DENIS PJ0255-47, 5 \\
\hline 2MASS J031854.0-342129 & L7 & 15.53 & 14.31 & 13.48 & 16.41 & 14.40 & $16.5 \pm 2.3$ & 6 \\
\hline 2MASS J044337.6+000205 & M9 & 12.52 & 11.80 & 11.17 & 13.13 & 11.78 & $16.2 \pm 2.1$ & 7 \\
\hline 2MASS J083008.3+482848 & L8 & 15.44 & 14.34 & 13.68 & 16.22 & 14.42 & $13.09 \pm 0.59$ & V04, 8 \\
\hline 2MASS J085925.4-194926 & L7: & 15.51 & 14.44 & 13.73 & 16.37 & 14.47 & $16.3 \pm 3.8$ & 4 \\
\hline 2MASS J102248.2+582545 & L1 & 13.50 & 12.64 & 12.16 & 14.16 & 12.70 & $19.9 \pm 2.6$ & 3 \\
\hline 2MASS J102552.3+321235 & L7: & 15.91: & 15.59: & 15.07 & 17.90 & 15.96 & $\sim 35$ & 9 \\
\hline 2MASS J104307.5+222523 & L8 & 15.95 & 14.75 & 13.99 & 16.80 & 14.79 & $17.2 \pm 2.4$ & 6 \\
\hline 2MASS J105847.8-154817 & L3 & 14.18 & 13.24 & 12.51 & 14.92 & 13.26 & $17.3 \pm 0.3$ & 10, D02 \\
\hline 2MASS J115539.5-372735 & L2 & 12.81 & 12.04 & 11.46 & 13.49 & 12.06 & $12.6 \pm 1.0$ & 11 \\
\hline 2MASS J120358.1+001550 & L4 & 14.01 & 13.06 & 12.48 & 14.74 & 13.10 & $15.2 \pm 1.5$ & 16 \\
\hline 2MASS J130042.5+191235 & L1 & 12.71 & 12.07 & 11.61 & 13.64 & 11.82 & $13.9 \pm 0.9$ & 2 \\
\hline 2MASS J142131.5+182741 & L0 & 13.23 & 12.43 & 11.94 & 13.83 & 12.46 & $20.0 \pm 1.2$ & 2,11 \\
\hline 2MASS J142528.0-365023 & L3 & 13.75 & 12.58 & 14.49 & 12.58 & 11.81 & $16.4 \pm 3.0$ & 12 \\
\hline 2MASS J143928.4+192915 & L1 & 12.76 & 12.04 & 11.55 & 13.41 & 12.07 & $14.37 \pm 0.10$ & D02, 13 \\
\hline 2MASS J150654.4+132106 & L3 & 13.41 & 12.41 & 11.75 & 14.05 & 12.40 & $14.1 \pm 1.3$ & 2 \\
\hline 2MASS J151500.9+484739 & L6 & 14.06 & 13.07 & 12.57 & 14.81 & 13.15 & $10.2 \pm 1.4$ & 4 \\
\hline 2MASS J172103.9+334415 & L3 & 13.58 & 12.92 & 12.47 & 14.31 & 12.99 & $15.2 \pm 1.4$ & 4 \\
\hline 2MASS J200250.7-052152 & L6 & 15.32 & 14.23 & 13.36 & 16.13 & 14.21 & $18.2 \pm 2.6$ & 6 \\
\hline 2MASS J202820.4+005227 & L3 & 14.30 & 12.38 & 12.79 & 15.00 & 13.41 & $21.1 \pm 2.0$ & 7 \\
\hline 2MASS J214816.3+400359 & L6.5 & 14.15 & 12.78 & 11.77 & 14.98 & 12.75 & $9.6 \pm 1.0$ & 3 \\
\hline 2MASS J223732.5+392239 & M9.5 & 13.35 & 12.68 & 12.15 & 13.98 & 12.68 & $18.9 \pm 0.7$ & 15, Hip \\
\hline
\end{tabular}

Notes. B07: Trigonometric parallax from Bartlett (2007); D02: Trigonometric parallax from Dahn et al. (2002); Hip: Trigonometric parallax from Hipparcos (ESA, 1997); T95: Trigonometric parallax from Tinney et al. (1995); V04: Trigonometric parallax from Vrba et al. (2004).

References. (1) Irwin et al. (1991); (2) Gizis et al. (2000); (3) Reid et al., in preparation (Paper X); (4) Cruz et al. (2003) (Paper V); (5) Martín et al. (1999); (6) Cruz et al. (2007) (Paper IX); (7) Hawley et al. (2002); (8) Geballe et al. (2002); (9) Chiu et al. (2006); (10) Delfosse et al. (1997); (11) Gizis (2002); (12) Kendall et al. (2004); (13) Kirkpatrick et al. (1999); (14) Kirkpatrick et al. (2000); (15) Kirkpatrick et al. (2001); (16) Fan et al. (2000).

Notes on individual stars. 2M1025+3212 has JHK photometry on the MKO system by Chiu et al. (2006); they list $J=16.89, H=15.98$, and $K=15.16$, corresponding to a spectroscopic distance of $\sim 35$ parsecs. $2 \mathrm{M} 2237+39$ is $\mathrm{G}$ 216-7B, a wide companion of an early-type M dwarf primary.

faint, with $M_{J} \sim 15.5$ for an L6 primary, and $M_{J} \sim 15.1$ for an L5 primary. In either case, the secondary is fainter than 2M2252-1730B, which has a spectral type of $\sim \mathrm{T} 2$ (Reid et al. $2006 \mathrm{~b}$ ) and is the coolest resolved secondary in an L/T system. This does not, however, necessarily imply a spectral type later than $\mathrm{T} 2$, since the $\left(M_{J}\right.$, spectral type) relation is double valued for dwarfs with spectral types in the range $\sim$ L5-T5 (see Figure 9, Kirkpatrick 2005).

The relative flux levels in the F110W and F170M filters provide the only constraints that we have on the spectral type of the secondary. The inferred color, $(J-H) \sim 1.00$, suggests that 2M2255-5713B has a spectral type in the range L7-T1 (see Figure 4 in Reid et al. 2006a). We have probed this issue further by constructing composite near-infrared spectra for the binary system. Besides matching the constraints offered by our imaging observations, this exercise probes the potential for discerning the nature of the secondary from ground-based spectroscopic observations.

Following the techniques described by Burgasser et al. (2007a), we have combined observations of spectral standards, fixing the relative flux levels to match the observed magnitude difference in F110W, $\Delta m_{110} \sim 1.5 \mathrm{mag}$. Figure 3 plots the most relevant spectral combinations. The results show that if the companion is an early $\mathrm{T}$ dwarf, we would not expect to see strong evidence for methane in the composite spectrum; at most, mild traces might be detectable at $2.2 \mu \mathrm{m}$. The observed flux ratio of 3.5:1 (or $\Delta m_{170} \sim 1.35 \mathrm{mag}$ ) at F170M favors an L5/L8 or L6/L8 system; the presence of methane absorption in the $\mathrm{T}$ dwarfs leads to higher flux ratios, $\sim 5: 1$ (or $\Delta m_{170} \sim 1.75 \mathrm{mag}$ ), in the mid-L+T combinations plotted in Figure 3 . We therefore conclude that $2 \mathrm{M} 2255-5713$ probably comprises a mid-L primary and a late-type L-dwarf secondary. Resolved spectroscopy of both components is required to fully characterize the system.

Using the $J$-band bolometric corrections from Reid et al. (2006a), and assuming a spectral type of L6 for the primary, we estimate luminosities of $M_{\text {bol }} \sim 15.4$ and $\sim 16.5$ for the primary and secondary. Figure 4 compares those estimates against theoretical mass-luminosity relations for ages $0.3-$ 5 Gyrs from Burrows et al. (1997) and Chabrier et al. (2000). The estimated mass of the primary ranges from $0.036 M_{\odot}$ at $\tau=0.3$ Gyrs to $0.075 M_{\odot}$ at 5 Gyrs, and the inferred mass ratio, $q$, ranges from 0.64 at 0.3 Gyrs to $q=0.95$ at $\tau=5$ Gyrs.

\section{DISCUSSION}

The prime goal of these observations is improving the estimate of the frequency of L-dwarf binary systems, where an "L-dwarf binary" is defined as a close binary with an Ldwarf primary. There are $85 \mathrm{~L}$ dwarfs in the current 20-parsec 

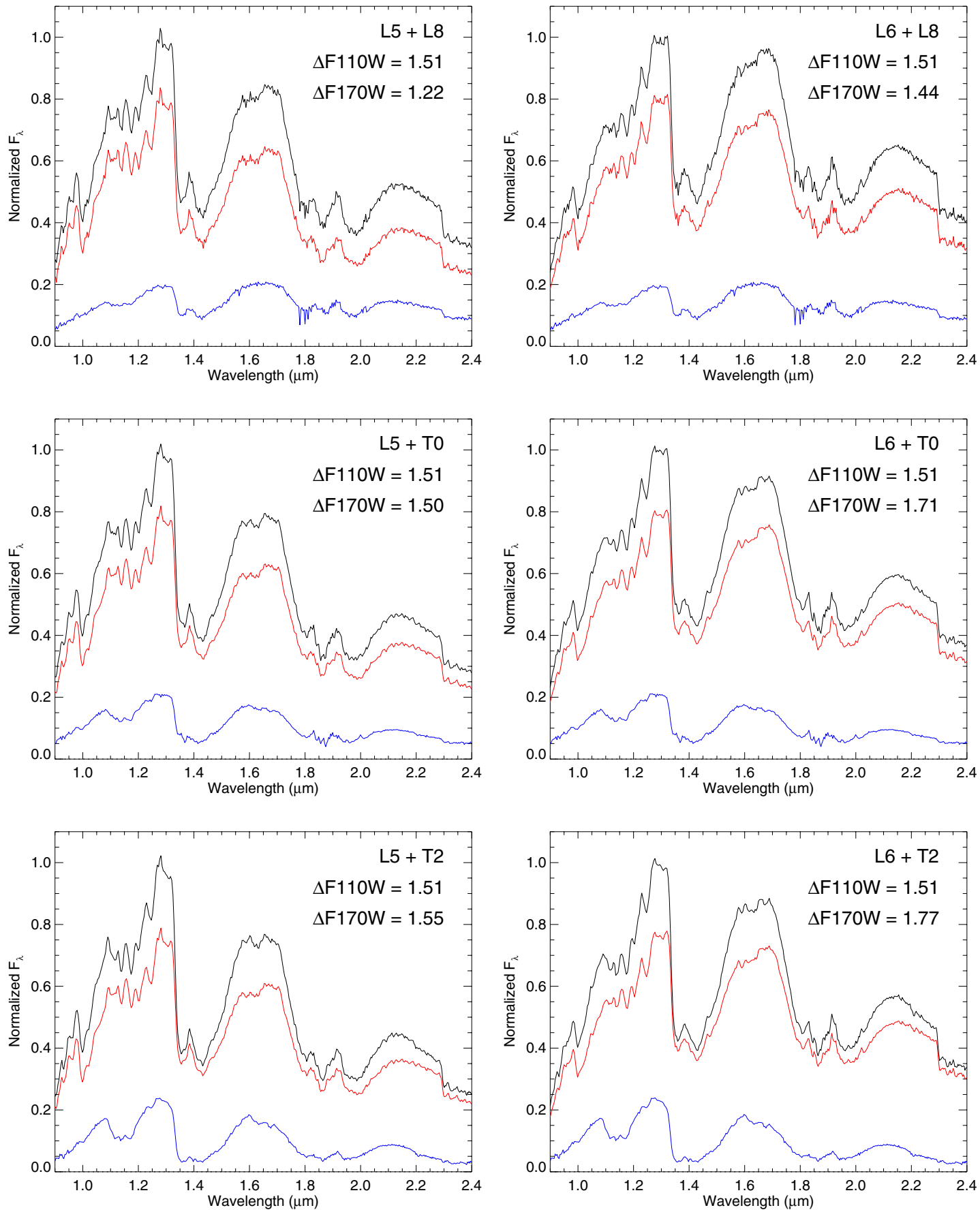

Figure 3. Composite spectra for late-L and late-L/early-T binaries. In each case, the primary spectrum is plotted in red, the secondary in blue and the composite spectrum in black. The primary and secondary spectra are scaled to match the observed flux ratio in the F110W filter.

census (Reid et al. 2007). The observations discussed in this paper include 20 unresolved L dwarfs with formal distance estimates $d \leqslant 20$ parsecs, and one binary system. Our previous NICMOS observing campaign (Reid et al. 2006b) covered $48 \mathrm{~L}$ dwarfs from our 20-parsec sample. Our observations resolved six as binaries, including one system, $2 \mathrm{M} 0025+4759$, lying beyond 20 parsecs. Our original distance estimate for another binary, 2M2152+0937, also placed it beyond 20 parsecs, but a revised spectral type of $\mathrm{L} 7$ brings it within the formal distance limits. The four other L-dwarf binaries are 2M0700+3157,
2M0915+0422, 2M2252-1730 and 2M0004-4044, The last system is LHS 102BC, an equal-mass L4.5/L4.5 wide companion of the M3.5 dwarf, LHS 102A.

Our original analysis placed 42 unresolved L dwarfs from Reid et al. (2006b) within 20 parsecs. However, we have reclassified one system (2M2351-2537) as an M8 dwarf, while spectral-type revisions for two other unresolved dwarfs $(2 \mathrm{M} 1428+5923$ and $2 \mathrm{M} 1552+2948)$ lead to revised distances that exceed 20 parsecs. Thus, our previous NICMOS observations encompass 39 unresolved and five binary L dwarfs from 


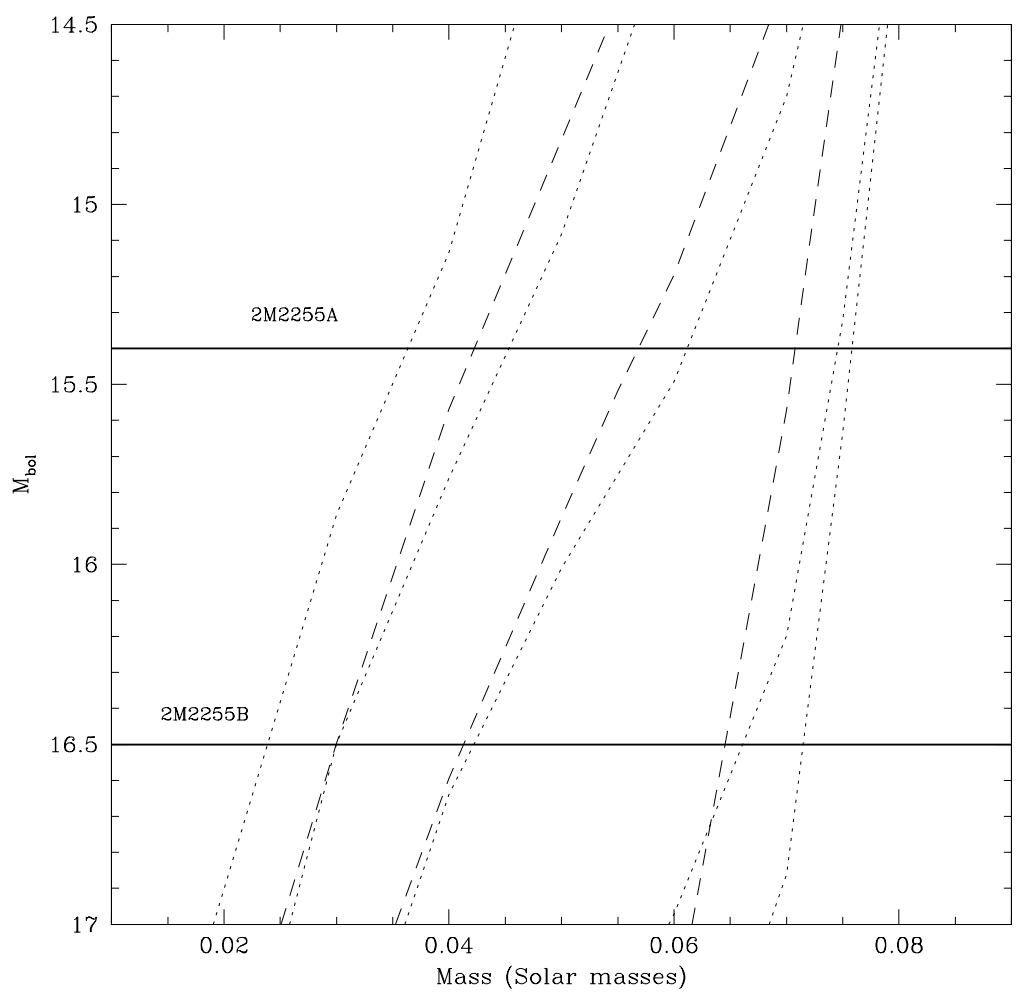

Figure 4. Constraining the mass ratio of 2M2255-5713: the dotted lines plot ( $M_{\mathrm{bol}}$, mass) isochrones from the Burrows et al. (1997) models for ages of 0.3, 0.5, 1, 3 , and 5 Gyrs (from left to right), while the dashed lines plot data for the Chabrier et al. (2000) "dusty" models for ages $0.5,1$, and 5 Gyrs. The horizontal lines mark the location of the two components of 2M2255-5713, assuming a spectral type of L6 for the primary.

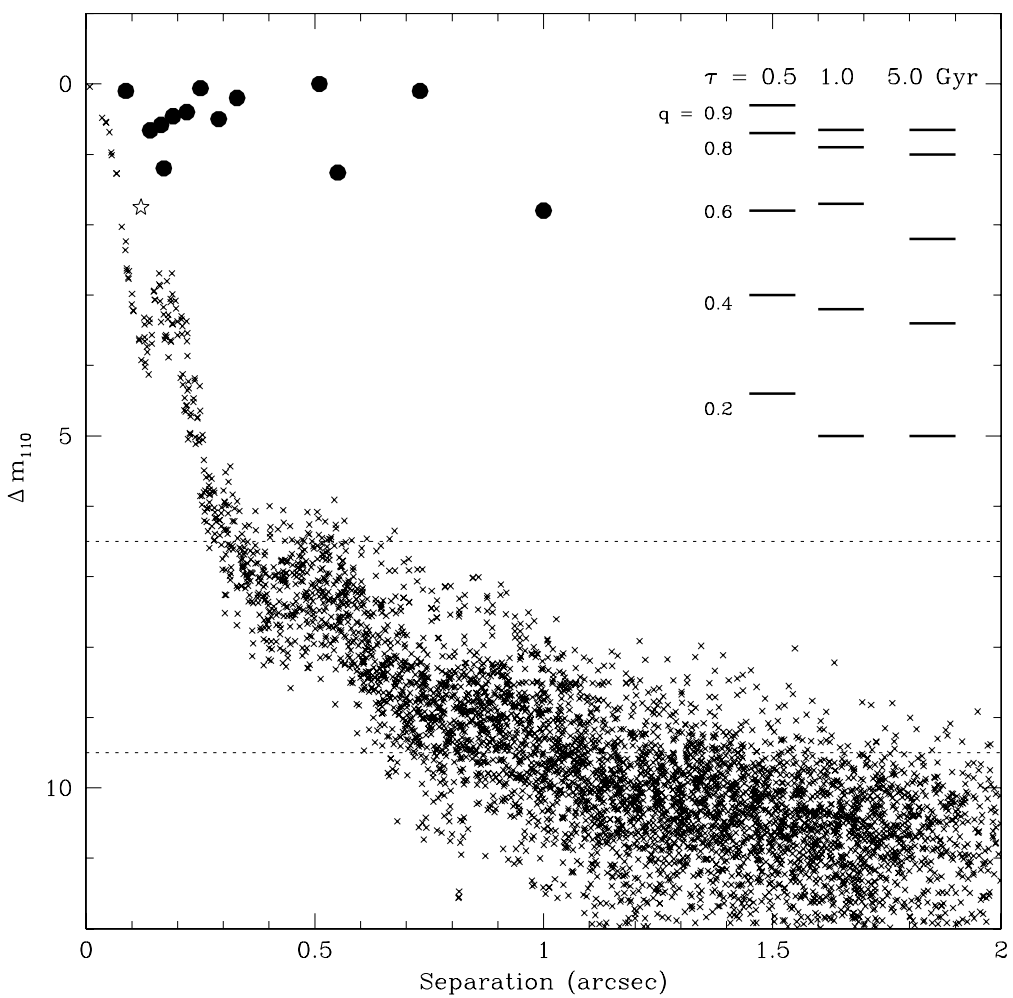

Figure 5. The F110W PSF for the L dwarf, 2M0523-1403, plotted in a magnitude scale, relative to the peak brightness. The solid points plot data for the nearby L-dwarf binaries discussed by Reid et al. (2006a); the 5-point star marks the location of 2M2255-5713. The dotted lines mark the range of effective detection limits set by the sky background; the lower line corresponds to a primary with $J \sim 13$, the upper corresponds to a primary with $J \sim 16$. The sequence of short horizontal lines indicates the magnitude difference predicted for a $0.07 M_{\odot}$ primary and a secondary with $0.9 \geqslant q \geqslant 0.2$ and ages $0.5,1.0$, and 5 Gyrs; these predictions are based on the model calculations by Burrows et al. (1997). 

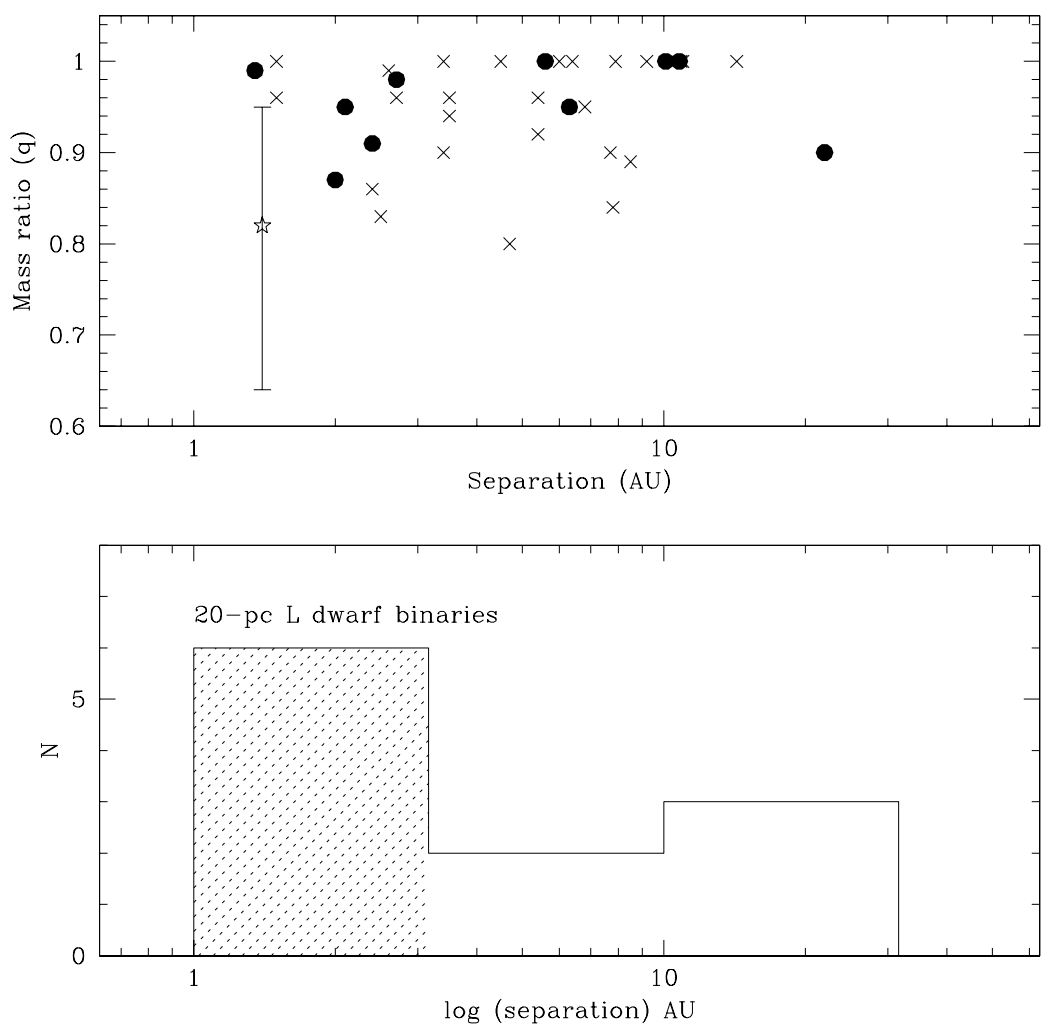

Figure 6. The upper panel plots the mass ratio as a function of separation, in astronomical units, for binary systems with L-dwarf primaries listed in Table 1 of Burgasser et al. (2006). The location of 2M2255-5713 is marked as a star, with the errorbars indicating the uncertainty in the mass ratio estimate; solid points identify other dwarfs in the 20-parsec sample; and the crosses are binaries beyond that distance limit; the widest binary is 2M1520-4422 (Burgasser et al. 2007b; Kendall et al. 2007). The lower panel shows the distribution of separations for the 11 L-dwarf binaries within 20 parsecs; the shaded section of the histogram is sampled incompletely by the current set of NICMOS imaging data.

the current 20-parsec sample. Combining those data with the present sample, we have HST NICMOS observations covering 65 L dwarf systems from the 20-parsec sample; we identify six binaries with L dwarf primaries and 59 unresolved L dwarfs.

A further four $\mathrm{L}$ dwarfs within 20 parsecs were known as binary systems prior to our initiating the NICMOS HST programs (Denis0205-1159, ${ }^{9}$ 2M0423-0414, 2M0746+2000, and Kelu 1; see Table 4 in Reid et al. 2006a). More recently, Burgasser et al. (2007b) and Kendall et al. (2007) have identified 2MASS J15200224-4422419AB as an L1.5+L4.5 binary at a distance of $19 \pm 2$. Finally, two L dwarfs, 2M0539-0059 and $2 \mathrm{M} 1632+1904$, are unresolved in I-band WFPC2 observations (Reid et al. 2001; Gizis et al. 2003). Another L dwarf, 2MASS J03105986+1648155, originally fell within the 20-parsec sample as a single L8 dwarf; however, Stumpf et al. (2005) have resolved it as a binary, and the revised spectroscopic parallax places it just beyond the 20-parsec limit. Thirteen L dwarfs in the 20-parsec census still lack high-resolution imaging. Considering the 72 systems with such data, we identify 11 binaries with $\mathrm{L}$ dwarf primaries and 61 unresolved $\mathrm{L}$ dwarfs. This corresponds to a formal binary fraction of $15.3_{-3.1}^{+5.1} \%$, where the $1 \sigma$ uncertainties are derived using the binomial formalism outlined by Burgasser et al. (2003).

Most L dwarfs in the current dataset have distances derived from spectroscopic parallaxes. Consequently, we should apply Malmquist corrections before deriving statistical parameters, such as the apparent binary frequency. The $M_{J} /$ spectral type

\footnotetext{
9 Bouy et al. (2005) have suggested that Denis0205-1159 is a triple system.
}

relation has a dispersion of \pm 0.35 mag (Cruz et al. 2003), corresponding to systematic absolute magnitude corrections of $-0.12 \mathrm{mag}$. This has the net effect of reducing the effective distance limit for statistical analysis to 19 parsecs. There are eight binaries and 56 unresolved $\mathrm{L}$ dwarfs within that distance limit, giving a frequency of $12.5_{-3.0}^{+5.3} \%$ for resolved binary systems.

The formal detection limits for our NICMOS observations are illustrated in Figure 5 (an updated version of Figure 6 from Reid et al. 2006a). The figure plots the radial profile (PSF) of an unresolved L dwarf (2M0523-1403, from our Cycle 13 dataset) in relative magnitudes; to be detected, a companion should have a peak brightness that lies significantly above the PSF at the appropriate separation. The known $\mathrm{L}$ dwarf companions are plotted at the appropriate relative magnitudes and separations. All lie well above the detection limit. We also plot the relative magnitudes predicted for binary systems with a $0.07 M_{\odot}$ primary and companions with mass ratios $0.2<q<0.9$ and $\tau=0.5$, 1.0 and 5.0 Gyrs. The predictions are based on the Burrows et al. (1997) set of low-mass star/brown dwarf models.

Figure 6 demonstrates that the NICMOS observations are capable of detecting binaries with mass ratios $q>0.6$ at separations $\Delta>0.15$ arcsec for systems older than $\tau=$ 1 Gyr. All L-dwarf binaries, regardless of age, with $q>0.2$ are detectable at $\Delta>0.3$ arcsec. The absence of such systems among the present sample argues either that such systems never form, or that they have short timescales against disruption.

Since we have distance estimates for each binary, we can estimate the component separation in absolute units. The upper 
panel in Figure 6 plots the separations of known L-dwarf binaries as a function of the mass ratio (data from Table 1 of Burgasser et al. 2007a), while the lower panel shows the separation distribution for the 11 binaries that are formally within 20 parsecs. The overwhelming majority of these systems are drawn from the field, and are therefore likely to be high-mass L dwarfs with ages exceeding $1 \mathrm{Gyr}$ and total masses between 0.12 and $0.15 M_{\odot}$. The statistics are sparse, but it is clear that the most systems lie at separations less than $3 \mathrm{AU}$, i.e. within the range that is sampled incompletely by imaging surveys.

A handful of ultracool binary systems have been identified at smaller separations, based either on spectroscopic peculiarities or radial velocity measurements. Cruz et al. (2004) demonstrated that the unusual near-infrared spectrum of 2MASS J05185995-2828372 is well matched by an unresolved L/T binary, and subsequent NICMOS observations have confirmed that the system is a binary (Burgasser et al. 2006). There have been suggestions that many early-type $\mathrm{T}$ dwarfs are unresolved $\mathrm{L} / \mathrm{T}$ binaries (Liu et al. 2006; Burgasser 2007a). SDSS J080531.84+481233.0 (Burgasser 2007b), which is not included in the present NICMOS sample, is the only such candidate within 20 parsecs.

Radial velocity surveys also probe binaries at smaller separations. Four brown dwarf spectroscopic binaries are currently known: the low luminosity Pleiad, PPl 15, (Basri \& Martín 1999); the eclipsing system, 2MASS J0535-0546 (Stassun et al. 2006); the (probable) triple brown dwarf, Gl 569B (Simon et al. 2006); and the recently discovered ChaH $\alpha 8$ (Joergens \& Müller 2008). Due to their relative youth, all are currently M dwarfs, although they will evolve through spectral class L and T. The first three are short-period systems, with near-equal mass ratios; in contrast, $\mathrm{ChaH} \alpha 8$ has $q<0.4$, a period of 1,590 days and a semi-major axis of $\sim 1 \mathrm{AU}$.

At the present juncture, there are relatively few high-precision radial velocity measurements for field $\mathrm{L}$ dwarfs. As a reference, a binary system with two $0.05 M_{\odot}$ components in a circular orbit, semi-major axis 1.0 AU, has a period of 0.9 years, and an orbital velocity of $\sim 15 \mathrm{~km} \mathrm{~s}^{-1}$. Such a binary system is readily detectable using currently available infrared spectrographs on large telescopes. Blake et al. (2007) have recently developed techniques for measuring velocities to significantly higher precision. So far, their observations are limited to short time baselines and nine nearby ultracool dwarfs, including seven L dwarfs from the 20-parsec sample. None show evidence for binary companions with periods less than 3 days, but it is clearly essential to compile more observations of a larger sample to probe binary frequency within the 3 AU inner boundary of the NICMOS observations.

\section{CONCLUSIONS}

We have used the NIC1 camera on HST to obtain highresolution near-infrared images of 27 nearby ultracool dwarfs, including $21 \mathrm{~L}$ dwarf systems within 20 parsecs of the Sun. One target, 2M2255-5713, is resolved as a binary, with a 4:1 flux ratio in the $\mathrm{F} 110 \mathrm{~W}$ passband. The primary has spectral type L5/L6, and the secondary is likely to have a spectral type between L8 and T1. Matching the inferred bolometric magnitudes against model predictions, we estimate that the mass ratio lies between 0.64 (for $\tau=0.3 \mathrm{Gyrs}$ ) and 0.95 ( $\tau=5$ Gyrs). The mass ratio exceeds 0.8 for an age $\tau>1$ Gyr. The remaining 27 dwarfs are unresolved.

Combining the present results with our earlier observations (Reid et al. 2006a) and literature data, we derive a formal frequency of $12.5_{-3.0}^{+5.3} \%$ for resolved binaries in the local L-dwarf population. Our observations are capable of detecting systems with mass ratios $q>0.2$, but all known systems are near-equal mass, suggesting that low- $q$ systems either fail to form, or have short lifetimes. The distribution of separations for the nine L-dwarf binaries known in the 20-parsec sample indicates that the binary frequency increases as separations decrease from 15 to $<3$ AU. Radial velocity monitoring can set constraints on binary frequency at smaller separations. No spectroscopic L-dwarf binaries are currently known, but more extensive monitoring of a larger subset of the local L dwarfs is required to map the full range of properties of ultracool binaries.

The observations described in this paper are associated with HST program \#10879, and those data were obtained via the HST data archive facilities maintained at the Space Telescope Science Institute. Support for this research was provided by NASA through a grant from the Space Telescope Science Institute, which is operated by the Association of Universities for Research in Astronomy, Inc., under NASA contract NAS 5-26555. K.L.C. is supported by an NSF Astronomy and Astrophysics Postdoctoral Fellowship under award AST-0401418. M.C.L. acknowledges support from NSF grant AST-0507833 and an Alfred P. Sloan Research Fellowship. Support for K.L.C. was provided by NASA through the Spitzer Space Telescope Fellowship Program, through a contract issued by the Jet Propulsion Laboratory, California Institute of Technology under a contract with NASA. This publication makes use of data from the 2 Micron All Sky Survey, which is a joint project of the University of Massachusetts and the Infrared Processing and Analysis Center, and funded by the National Aeronautics and Space Administration and the National Science Foundation. 2MASS data were obtained from the NASA/IPAC Infrared Science Archive, which is operated by the Jet Propulsion Laboratory, California Institute of Technology, under contract with the National Aeronautics and Space Administration.

\section{REFERENCES}

Basri, G., \& Martín, E. L. 1999, ApJ, 118, 2460

Bartlett, J. L. 2007, PASP, 119, 828

Blake, C. H., Charbonneau, D., White, R. J., Marley, M. S., \& Saumon, D. 2007, ApJ, 666, 1198

Bouy, H., Martín, E. L., Brandner, W., \& Bouvier, J. 2005, AJ, 129, 511

Burgasser, A. J. 2007a, ApJ, 659, 655

Burgasser, A. J. 2007b, AJ, 134, 1330

Burgasser, A. J., Kirkpatrick, J. D., Cruz, K. L., Reid, I. N., Leggett, S. K. Liebert, J., Burrows, A., \& Brown, M. E. 2006, ApJS, 166, 585

Burgasser, A. J., Kirkpatrick, J. D., Reid, I. N., Brown, M. E., Miskey, C. L., \& Gizis, J. E. 2003, ApJ, 586, 512

Burgasser, A. J., Looper, D. L., Kirkpatrick, J. D., \& Liu, M. C. 2007b, ApJ, 658,557

Burgasser, A. J., Reid, I. N., Siegler, N., Close, L. M., Allen, P., Lowrance, P. J., \& Gizis, J. E. 2007a, in Planets and Protostars V, ed. B. Reipurth, D. Jewitt, \& K. Keil (Tucson, AZ: Univ. Arizona Press), 427

Burrows, A., et al. 1997, ApJ, 491, 856

Chabrier, G., Baraffe, I., Allard, F., \& Hauschildt, P. 2000, ApJ, 542, 464

Chiu, K., Fan, X., Leggett, S. K., Golimowski, D. A., Zheng, W., Geballe, T. R., Schneider, D. P., \& Brinkmann, J. 2006, AJ, 131, 2722

Close, L. M., Siegler, N., Freed, M., \& Biller, B. 2003, ApJ, 587, 407

Cruz, K. L., Burgasser, A. J., Reid, I. N., \& Liebert, J. 2004, ApJ, 604, L61

Cruz, K. L., Reid, I. N., Liebert, J., Kirkpatrick, J. D., \& Lowrance, P. J. 2003, AJ, 126,2421

Cruz, K. L., Reid, I. N., Liebert, J., Kirkpatrick, J. D., \& Lowrance, P. J. 2007, AJ, 133,439

Dahn, C. C., et al. 2002, AJ, 124, 1170

Delfosse, X., et al. 1997, A\&A, 327, L25 
Duquennoy, A., \& Mayor, M. 1991, A\&A, 248, 485

Fan, X., et al. 2000, AJ, 119, 928

Fischer, D. A., \& Marcy, G. W. 1992, ApJ, 396, 178

Geballe, T. R., et al. 2002, ApJ, 564, 466

Gizis, J. E. 2002, ApJ, 575, 484

Gizis, J. E., Monet, D. G., Reid, I. N., Kirkpatrick, J. D., Liebert, J., \& Williams, R. J. 2000, AJ, 120, 1085

Gizis, J. E., Reid, I. N., Knapp, G. R., Liebert, J., Kirkpatrick, J. D., Koerner, D. W., \& Burgasser, A. J. 2003, AJ, 125, 3302

Hawley, S. L., et al. 2002, AJ, 123, 3409

Irwin, M., McMahon, R. G., \& Reid, N. 1991, MNRAS, 252, 61P

Joergens, V., \& Müller, A. 2008, A\&A, in press

Kendall, T. R., Delfosse, X., Martín, E. L., \& Forveille, T. 2004, A\&A, 416, L17

Kendall, T. R., Jones, H. R. A., Pinfield, D. J., Pokorny, R. S., Folkes, S., Weights, D., Jenkins, J. S., \& Mauron, N. 2007, MNRAS, 374, 445

Kirkpatrick, J. D. 2005, ARA\&A, 43, 195

Kirkpatrick, J. D., Henry, T. J., \& Irwin, M. J. 1997, AJ, 113, 142

Kirkpatrick, J. D., Liebert, J., Cruz, K. L., Gizis, J. E., \& Reid, I. N. 2001, PASP, 113,814
Kirkpatrick, J. D., et al. 1999, ApJ, 519, 802

Kirkpatrick, J. D., et al. 2000, AJ, 120, 447

Liu, M. C., Leggett, S. K., Golimowski, D. A., Chiu, K., Fan, X. Geballe, T. R., Schneider, D. P., \& Brinkmann, J. 2006, ApJ, 647, 1393

Martín, E. L., Brandner, W., \& Basri, G. 1999, Science, 283, 1718

Reid, I. N., Cruz, K. L., Allen, P. R., Mungall, F., Liebert, J., \& Lowrance, P. 2007, AJ, submitted

Reid, I. N., Gizis, J. E., Kirkpatrick, J. D., \& Koerner, D. W. 2001, AJ, 121, 489 Reid, I. N., Lewitus, E., Allen, P. R., Cruz, K. L., \& Burgasser, A. J. 2006a, AJ, 132,891

Reid, I. N., Lewitus, E., Burgasser, A. J., \& Cruz, K. L. 2006b, ApJ, 639, 1114 Schmidt, S. J., Cruz, K. L., Bongiorno, B. J., Liebert, J., \& Reid, I. N. 2007, AJ, 133,2258

Simon, M., Bender, C., \& Prato, L. 2006, ApJ, 644, 1183

Skrutskie, M. F., et al. 2006, AJ, 131, 1163

Stassun, K. G., Mathieu, R. D., \& Valenti, J. 2006, Nature, 440, 311

Stumpf, M. B., Brandner, W., \& Henning, T. 2005, Protostars and Planets V, 8571

Tinney, C. G., Reid, I. N., Gizis, J., \& Mould, J. R. 1995, AJ, 110, 3014

Vrba, F. J., et al. 2004, AJ, 127, 2948 